\title{
Envejecimiento y alta prevalencia de estadíos avanzados determinan la creciente mortalidad por cáncer mamario en Chile*
}

\author{
Drs. IVÁN SERRA C. ${ }^{1}$, RAÚL MARTÍNEZ R. ${ }^{2}$, Sra. GLORIA REYES C. ${ }^{3}$, \\ Al. PAULA SIERRA V. ${ }^{4}$, Dra. CLAUDIA AGUAYO S. ${ }^{5}$ \\ 1 Servicios de Cirugía y Urgencia, Hospital Sótero del Río, Santiago. \\ 2 Servicio de Cirugía, Hospital Sótero del Río, Santiago. \\ 3 Estadístico, Departamento de Estadística e Informaciones en Salud. Ministerio de Salud (DEIS), Santiago. \\ 4 Alumna de Medicina. Pontificia Universidad Católica de Chile, Santiago. \\ 5 Epidemióloga, Hospital Regional de Concepción, Concepción. \\ Chile.
}

\begin{abstract}
Aging and high prevalence of advanced stages determine the increasing breast cancer mortality in Chile
\end{abstract}

Aims: To test the hypothesis that ages above 54 years not included in the national program and a greater proportion of advanced stages in the public health sector that attends $75 \%$ of the population, could explain the increasing high mortality rate for this cancer in Chile. Methods: Incidence data provided by the 29 public health services (SNSS) in the country and mortality data from the Ministry of Health were used to analyse the epidemiological situation at the biggest health service (SSMSO), 1.6 million of people, compared with the country, 16 million. Results: Breast cancer was in 2009, last year with information, the first cause of oncologic death in Chilean women with 1,338 deaths and an actual mortality rate of 15.7 per 100,000 women. Deaths for gallbladder and gastric cancers were 1,295 and 1,150 respectively. Mortality for breast cancer in 2009 was 10.4 in the SSMSO. Less advanced stages with a proportion of about $20 \%$ compared with the country (30\%) and more prevalent older ages were observed in this health service. Conclusions: Hypothesis is true. Targets of extended age and decreasing of less advanced stages for the country emerge from this study. To integrate levels of medical care in the SNSS and to include examination of breasts jointly with free diagnostic mammographies in women above 54 years old ordered by the nurses responsibles at the national programs of diabetes mellitus and arterial hypertension are recommended aiming to increase compliance of secondary prevention in breast cancer.

Key words: Breast cancer, epidemiology, incidence, mortality, health programs, Chile.

*Recibido el 26 de septiembre de 2011 y aceptado para publicación el 12 de diciembre de 2011.

Correspondencia: Dr. Iván Serra C.

Concha y Toro 3459, Santiago, Chile.

ivan.serra.canales@gmail.com 


\section{Resumen}

Objetivos: Es un estudio retrospectivo, descriptivo y analítico sobre cáncer de mama comparando incidencia nacional, sector público (SNSS) y mortalidad (Chile), últimas dos décadas, con la del Servicio de Salud Sur Oriente de Santiago (SSMSO). La hipótesis de mayores edades y menor frecuencia de estadíos avanzados en el SSMSO explicaría su menor mortalidad. Material y Método: Se utilizan datos de mortalidad del Ministerio de Salud y de incidencia aportados por los 29 Servicios de Salud del país. Resultados: Cáncer de mama aparece el 2009 como primera causa de mortalidad oncológica en la mujer chilena, con 1.338 muertes y tasa de 15,7 por 100.000 mujeres, superando al vesicular con 1.295 y al gástrico con 1.115. Las tasas de mortalidad por cáncer mamario en Chile fueron 12,1; 13,2 y 15,7, años 1991, 2000 y 2009 mientras en el SSMSO fueron más bajas: 11,4; 12,7 y 10,4 en esos años. Entre 30 y 34\% de las pacientes diagnosticadas del SNSS tenía más de 64 años comparado con el SSMSO, donde predominaron con más del $40 \%$ sobre 60 años, entre 1999 y 2009. Los estadíos avanzados, III y IV, presentaron una proporción estable 2001-2009, promedio 29,4\% en el SNSS, mientras bajó progresivamente en el SSMSO hasta 18,3\% el 2007. Discusión y Conclusiones: Se comprueba hipótesis ya que puede atribuirse la menor mortalidad por este cáncer en el SSMSO a menores tasas de estadíos avanzados. Este estudio permite proponer edades sobre 54 años para la pesquisa con mamografía diagnóstica. Además, integrar los niveles de atención médica: primario, secundario, terciario y extender pesquisa a mujeres mayores en programas de hipertensión arterial y diabetes mellitus, incorporando a sus enfermeras.

Palabras clave: Cáncer de mama, epidemiología, incidencia, mortalidad, programas de salud, Chile.

\section{Introducción}

El cáncer de mama como causa de muerte oncológica en mujeres es de alta frecuencia en todo el mundo, especialmente en los países desarrollados ${ }^{1,2}$. En Chile ha ocupado el segundo lugar desde el año 2000, en el cual, con 1.027 muertes por esta causa, desplazó al gástrico que tuvo 1.012, siendo ambas localizaciones superadas por el de origen vesicular que registró el mismo año 1.363 fallecimientos femeninos, manteniendo el primer lugar que ocupaba desde $1986^{3}$.

Posteriormente, el cáncer vesicular ha comenzado a disminuir en forma moderada y el mamario a aumentar en forma constante, además de muy marcadamente en los últimos años. Este hecho se hace inaparente al publicarse, a veces, sólo tasas ajustadas por edad en vez de observadas o crudas ${ }^{4}$. Desde el punto de vista de atención médica, oferta de servicios y solución de la demanda, las observadas son las más relevantes.

El aumento de la mortalidad por cáncer de mama en nuestro país se contrapone con el concepto errado de que la proporción de estadíos avanzados, III y IV, estaría disminuyendo en el sector público de salud chileno. Por supuesto, si esto último fuera verdadero constituiría una paradoja. La realidad es muy diferente ya que lo real no publicado es que el 30\% anual observado desde el año 2001 es bastante estable en el nivel nacional hasta la fecha. Esta información es entregada anualmente por los servicios de salud a la unidad correspondiente del Ministerio de Salud. Por falta de difusión, a pesar de que su promedio 20002008 fue estable de $29,6 \%$ y el 2000-2009 de 29,4\%, no hay debate en la materia y se promueve la idea errónea de que estamos disminuyendo los estadíos avanzados y además controlando la enfermedad.

El hecho de alta proporción mantenida y sin mejoría de los estadíos avanzados de cáncer de mama en el sistema nacional de servicios de salud (SNSS) explica, junto con el envejecimiento poblacional y elevadas tasas de mortalidad en senescentes, la alta y creciente mortalidad por este cáncer ${ }^{5-7}$. El ajuste por edad muestra una mortalidad estable ${ }^{8}$.

La mortalidad es muy diferente según la edad que se considere ya que es el principal factor de riesgo, fuera de la condición de ser mujer. El año 2009 murieron en Chile 300 mujeres de 80 y más años por cáncer de mama (sobre 194.700 de mujeres de esa edad ese año) comparado con sólo 60 mujeres entre 40 y 44 años (sobre 622.000 mujeres de esa edad el 2009) ${ }^{3}$. Por supuesto, esos cánceres se iniciaron clínicamente en ambos grupos etarios con anterioridad al fallecimiento pero ese intervalo entre diagnóstico y muerte no está bien establecido aunque se sabe que en pacientes con estadíos III y IV tiende a ser corto, inferior a cinco años ${ }^{9}$. Incluso, un estudio reciente en nuestro país muestra para universos 1997 y 2007 en un hospital de Santiago que estas pacientes fallecen en alta proporción antes de tres años ${ }^{7}$.

El objetivo de este estudio es describir la situación epidemiológica actual del cáncer de mama, enfatizando el importante aumento de su mortalidad en Chile en la última década. Al mismo tiempo, explorar las causas de dicho persistente incremento y fundamentar propuestas para su control.

\section{Material y Método}

Por carecer nuestro país de un registro nacional de cáncer, se ha utilizado en relación a incidencia 
en este estudio sólo la información referida al sector público chileno (SNSS). Los datos incluyen no sólo a beneficiarios del Fondo Nacional de Salud (Fonasa) sino también a no beneficiarios pero que se atienden en los establecimientos públicos de los tres niveles, primario, secundario y terciario. En consecuencia, hemos excluido por falta de información a los beneficiarios de las aseguradoras privadas (Isapres), de Fuerzas Armadas y de los servicios delegados, los cuales en conjunto pueden estimarse que constituyen un 22 a $25 \%$ del total de la población chilena ${ }^{9}$.

Se ha utilizado como única fuente de datos existente sobre incidencia de cáncer de mama en el sector público chileno, la información entregada anualmente por los 29 Servicios de Salud al nivel central (Unidad de Cáncer).

En relación a mortalidad se dispone de las estadísticas desarrolladas por el Departamento de Estadísticas en Salud (DEIS) publicadas periódicamente por el Ministerio en su página web. La información disponible 1990-2009, o sea, un período de 20 años es muy completa y presenta tasas crudas y ajustadas por edad, ambas anuales, por grupos quinquenales de edad y también las poblaciones correspondientes.

Como un patrón de comparación con el nivel nacional se ha utilizado la información existente tanto para incidencia como para mortalidad del SSMSO, donde se dispone de un registro poblacional incluyendo los datos hospitalarios del Sótero del Río y del Padre Hurtado. Son 1.485 pacientes entre 1994 y $2005^{5}$, que ampliado hasta el 2010 significan 2.775 mujeres con cáncer de mama, atendidas, tratadas y seguidas en su totalidad, con 100\% de verificación histopatológica.

\section{Resultados}

La mortalidad observada para este cáncer en Chile fue estable en la década del 90, entre 12 y 13 por 100.000 mujeres, comenzando a aumentar en la década del 2000 con incremento más marcado en los últimos años. Su ajuste por edad muestra estabilidad con tendencia moderada a la disminución (Figura 1).

Si se compara la magnitud de las muertes por este cáncer con los otros dos relevantes en la población femenina, se observa que a partir del 2001 el mamario superó al gástrico, 1.024 fallecimientos versus 967. Su incremento posterior y una leve disminución de las muertes por cáncer vesicular han hecho que el 2009, las muertes por cáncer de mama superen al anterior, 1.339 versus 1.295, manteniendo el gástrico la tercera posición con 1.115 decesos. En otras palabras, actualmente el cáncer de mama ocupa el primer lugar como causa de muerte oncológica femenina en Chile (Figura 2).

El análisis de las muertes según grupos etarios decenales permite observar que las mujeres más jóvenes significaron en Chile una proporción decreciente en las muertes por esta causa. El grupo de 30 a 39 años representó en 1991 un 7\%, el 2000 un 4\% y el 2009, sólo un 3\%. Entre 40 y 49 años descienden proporcionalmente desde un 18\% en 1991 a un 13\%

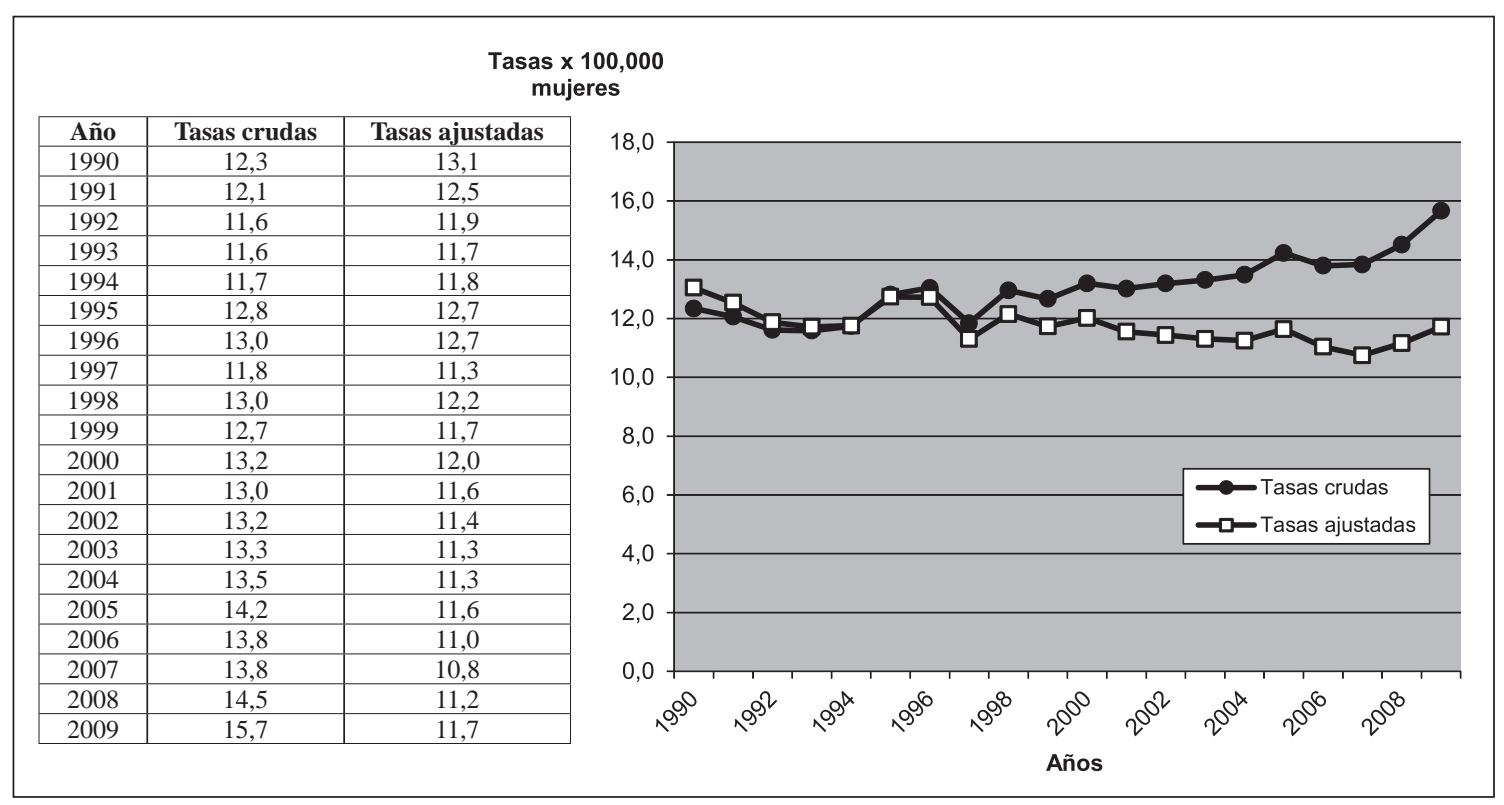

Figura 1. Tasas de mortalidad crudas y ajustadas por edad en cáncer de mama, Chile 1990-2009. 


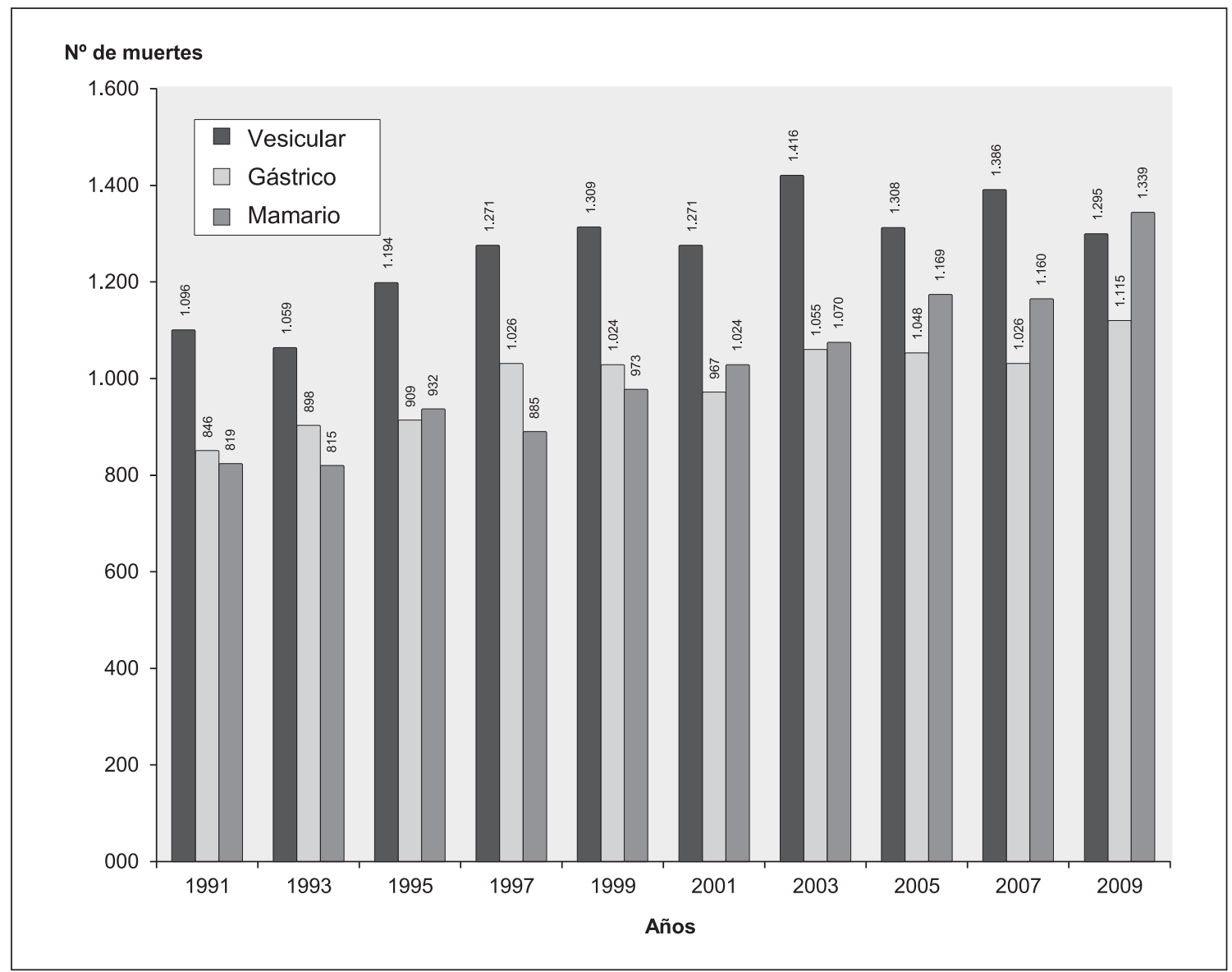

Figura 2. Evolución del número de muertes por cánceres vesicular, gástrico y mamario en mujeres, Chile 1991-2009.

el 2000, porcentaje que se mantiene el 2009. En cambio, las mujeres de 80 años y más, significaron en 1991 un 18\%, el 2000 un 20\%, y el 2009 un 18\% de las muertes por cáncer mamario. El grupo de 70 a 79 años mantiene proporciones entre 18 y $20 \%$. Los otros grupos no muestran grandes variaciones en el tiempo. Obviamente, las proporciones señaladas corresponden a magnitudes crecientes de muertes, lo que es más relevante en las mujeres de edades avanzadas (Figura 3).

El servicio más grande del país (SSMSO), con una de las tasas de mortalidad por este cáncer más bajas, muestra que las muertes en mujeres de 30 a 39 años disminuyen su importancia relativa de 4 a $2 \%$, mientras entre 40 y 49 aumentan en forma importante, desde 12 a 21\%. En cambio, a diferencia del promedio país, las muertes en pacientes de 70 a 79 años aumentan su importancia relativa desde 9 a 12\% (bastante menos que 18-20\%), y las de 80 años y más desde 11\% a 15\% (también menos que 18-20\%). El número total de muertes aumenta marcadamente en la década del 90 (de 47 a 86), pero en la última década, presenta un incremento bastante moderado (de 86 a 97), lo que se refleja en una mortalidad global de sólo 10,4 por 100.000 mujeres el 2009, más de un 50\% inferior a la nacional de 15,7 (Figura 4).

La estructura etaria de sobre 24.000 mujeres con cáncer de mama confirmado y tratadas en los 29 servicios de salud del país, período 1999-2009, ha sido consolidado por la unidad de cáncer del Ministerio en sólo tres grupos de edad: menores de 35 años, entre 35 y 64 y sobre 64. Por supuesto, la mayoría de los casos se observa en el grupo intermedio de 35 a 64 años, con dos tercios del total, pero que se producen en una población de 3,2 millones de mujeres, cuatro veces superior al grupo de 65 años y más. Este grupo, a pesar de constituir sólo un tercio de los casos nuevos en dicho período, corresponde a una población femenina de 800.000 personas. En consecuencia, su tasa resulta el doble de la correspondiente al grupo de 35 a 64 años. Bajo 35 años es sólo el 2\% (Figura 5). 


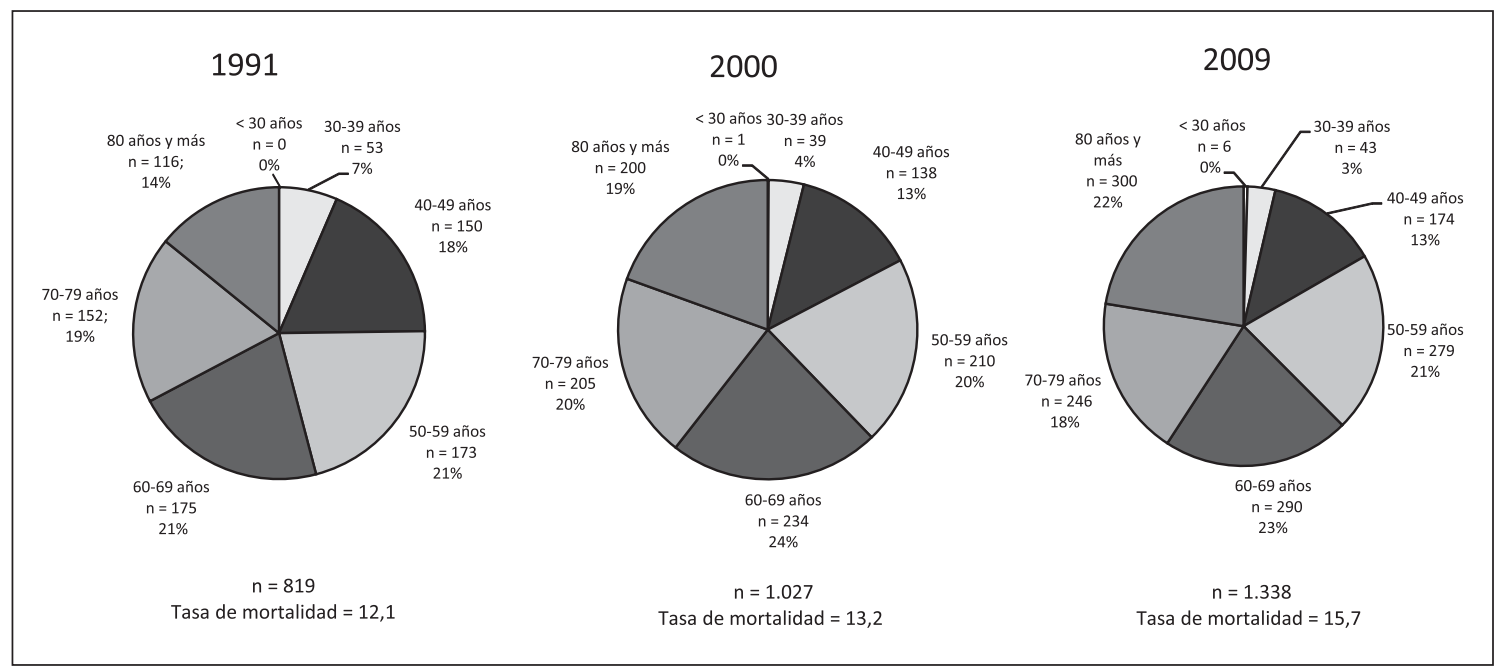

Figura 3. Número y proporción de fallecidas por cáncer de mama según edad (7 grupos etarios decenales) Chile 19912009.

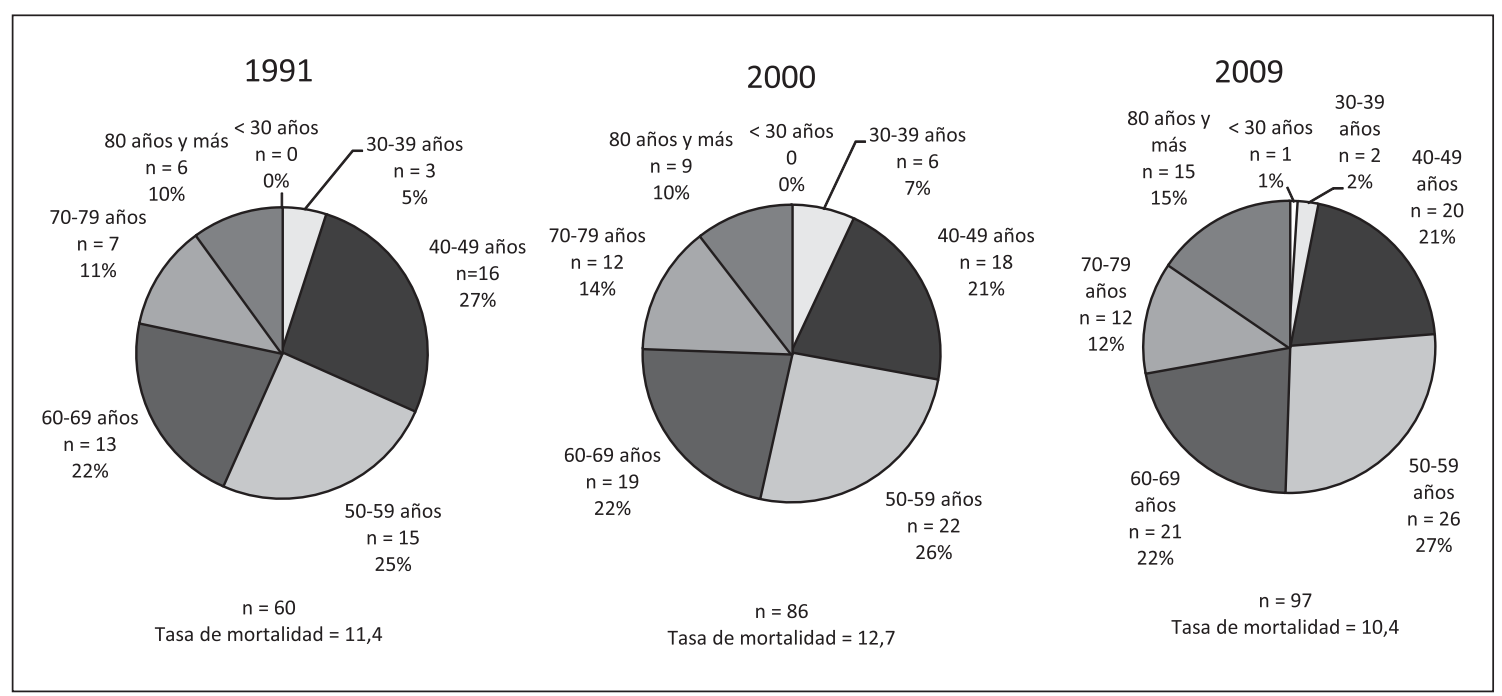

Figura 4. Número y proporción de fallecidas por cáncer de mama según edad (7 grupos etarios decenales) SSMSO 1991-2009.

La edad de los cánceres mamarios incidentes del SSMSO, separados en siete grupos etarios decenales, al compararse con lo nacional presenta importantes diferencias: las pacientes se concentran sobre 50 años (que tienen más alta mortalidad) e incluye magnitudes significativas sobre 60, 70 e incluso 80, con tasas aún mayores (Figura 6).

Sin embargo, tanto o más importante que la edad en los casos incidentes de cáncer de mama del SNSS, período 1999-2008, es la estadificación de las casi 25.000 pacientes diagnosticadas. La agrupación demasiado grande y diversa de etapas 0, I, II A y II
B, con una proporción de 43\% y 47\% en 1999 y 2000, sube a magnitudes entre 63 y 72\% del 2001 al 2008, sin diferenciar entre estas etapas. Al mismo tiempo, el conjunto de etapas III y IV baja desde 57 y 53\% en 1999 y 2000 a 30,8\% el 2001, con cifras entre 35 y 28\% desde el 2002, magnitud mantenida hasta el 2009. En otras palabras, el importante aumento en la década del 2000 de los cánceres más tempranos (0, I, II A, II B) no se ha acompañado en el SNSS de una disminución concomitante de los estadíos avanzados III y IV (Figura 7).

Los grados de avance en las pacientes incidentes 


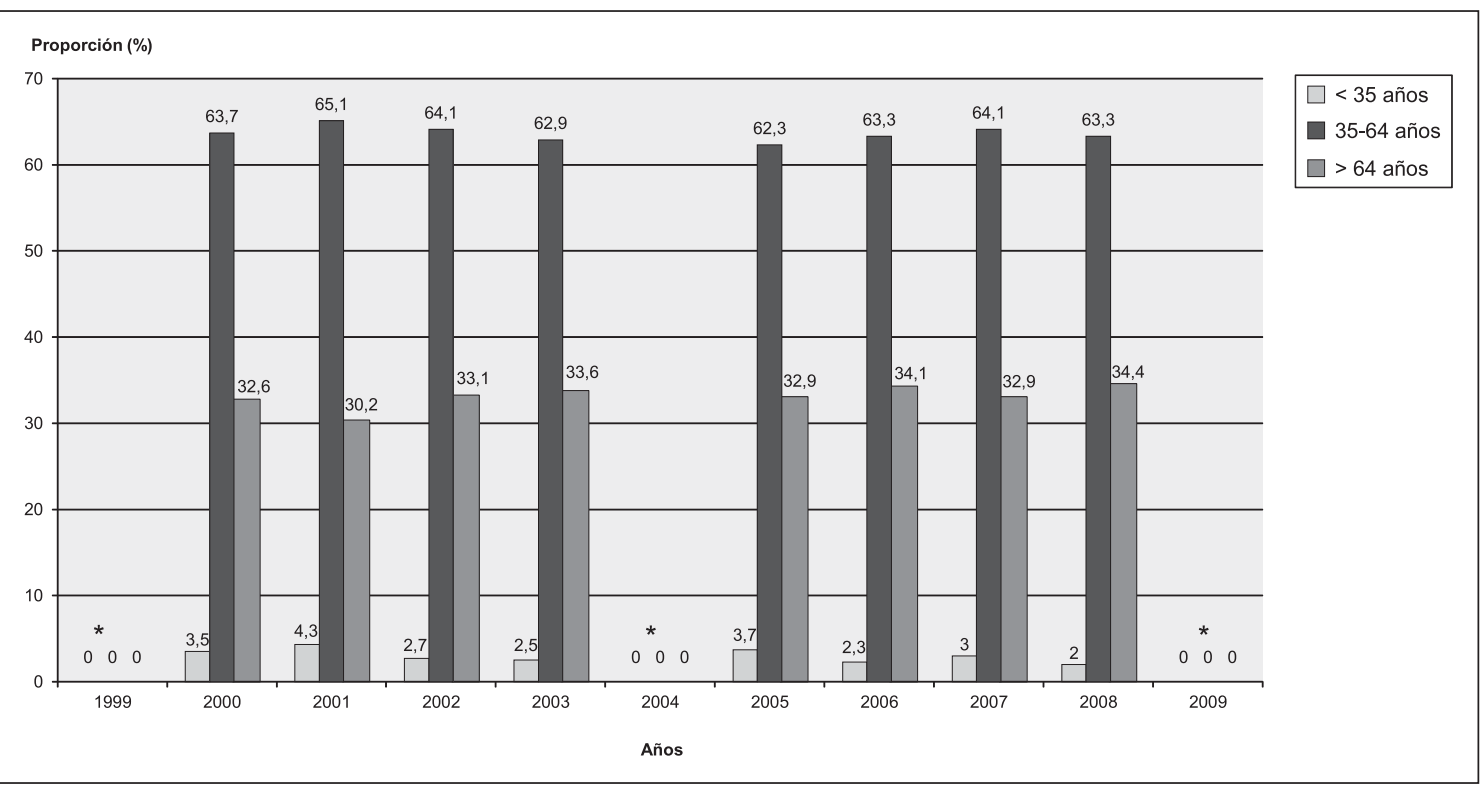

Figura 5. Incidencia proporcional de cáncer de mama según edad (3 grupos etarios) Chile (SNSS) 2000-2008. Nota: Ignorados 0,3-1\% según año considerado. Años 1999, 2004 y 2009, sin información.

del SSMSO muestran significativas menores tasas en comparación con el nivel nacional, además de estar mucho más desglosados. No es posible comparar los estadíos 0, I, II A y II B por la falta de diferenciación señalada. Pero los 0, I y II A entre 1994 y 2010 aumentan moderadamente en el SSMSO mientras los II B disminuyen marcadamente, de 24 a 17\% (datos no mostrados). Sin embargo, aún más importante es la disminución en el SSMSO de la proporción y cantidad de estadíos III y IV desde sobre $50 \%$ hasta bajo $20 \%$ el 2007, aunque posteriormente tiende a aumentar algo, pero siempre bajo el promedio país (Figura 8).

\section{Discusión}

Este estudio es parte de una línea de investigación sobre cáncer de mama iniciada hace cuatro años que busca aportar para el cumplimiento del objetivo del programa de reducir su mortalidad ${ }^{5-7}$.

El cáncer de mama en Chile ha sido la segunda causa de muerte oncológica en la mujer chilena, superando al gástrico desde el 2000. El primer lugar ha correspondido desde 1986 al vesicular y de vías biliares, en conjunto ${ }^{11}$, incluyendo este último el no especificado, el cual casi siempre se origina en la vesícula biliar ${ }^{12}$. El cáncer vesicular ha estado disminuyendo en la década del $2000^{13}$ y el de mama aumentando en forma constante, especialmente los últimos años ${ }^{5}$. Estos dos hechos han llevado a que el cáncer de mama ocupe el 2009, con 1.338 muertes y una tasa cruda de 15,7 por 100.000 mujeres, el primer lugar como causa de muerte oncológica en la mujer chilena, superando al vesicular y al gástrico en ese orden, con estos dos cánceres presentando 1.298 y 1.100 fallecimientos femeninos ${ }^{3}$.

El aumento notable de la mortalidad cruda del cáncer mamario con relativa estabilidad de la misma ajustada por edad, se explicaría por el envejecimiento poblacional y por el sesgo de recibir y tratar en hospitales públicos a mujeres relativamente jóvenes, preferentemente entre 40 y 50 años de edad ${ }^{14}$.

Es posible que el desconocimiento de la incidencia de cáncer de mama sin desglose por etapas en el SNSS sea causa o haya influido en una menor preocupación o inquietud sobre la falta de control de este cáncer en nuestro país. Se ha ignorado, en alguna medida, el aumento de su mortalidad, la importancia del envejecimiento poblacional, las enormes tasas de mortalidad en senescentes y especialmente, la persistencia durante toda la década del 2000 de una mantenida alta proporción de estadíos avanzados, III y IV, bordeando en el sector público de salud el $30 \%$.

La mortalidad por este cáncer en el SSMSO, crecientemente más baja en comparación con lo nacional, muy evidente en los grupos sobre 70 años, sugiere fuertemente que se debería a su captación preferente de mujeres sobre 50 años y bastante mayores, mientras en el SNSS tiende a privilegiar mujeres entre 40 y 50 años. Asociado a este diferencial mantenido e incrementado en los 20 años del 


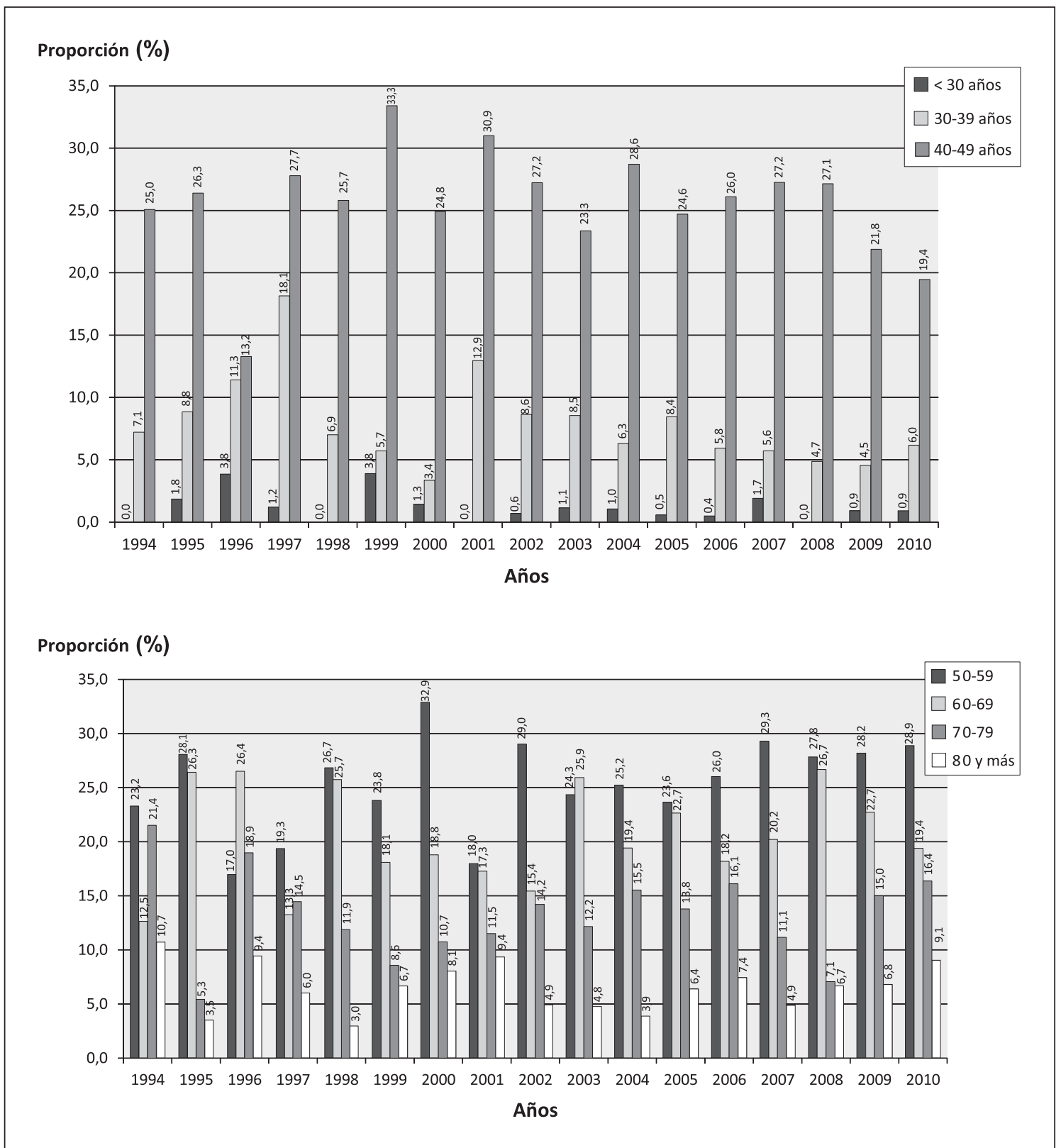

Figura 6. Incidencia proporcional de cáncer de mama según edad (7 grupos etarios) SSMSO 1994-2010.

período 1990-2009 aparece su menor proporción de estadíos avanzados III y IV como otra causa que explica el buen resultado sanitario del SSMSO. Esta proporción bajó a incluso menos de $20 \%$ el 2007 mientras en el SNSS ha estado permanentemente en casi 30\% en todo el período de nueve años, 20012009. La disminución brusca de la proporción de estos estadíos en el SNSS desde casi $60 \%$ en 1999 a 31\% el 2001 puede corresponder a un artefacto estadístico ya que por ser el cáncer una enfermedad crónica no es susceptible de cambios tan bruscos e importantes.

La favorable situación del SSMSO en cáncer de mama podría explicarse por la integración permanente de los especialistas hospitalarios con la atención primaria ${ }^{6}$. El caso chileno de aumento importante de estadíos 0 en el SNSS (dato no mostrado) sin la correlación esperable de disminución de 


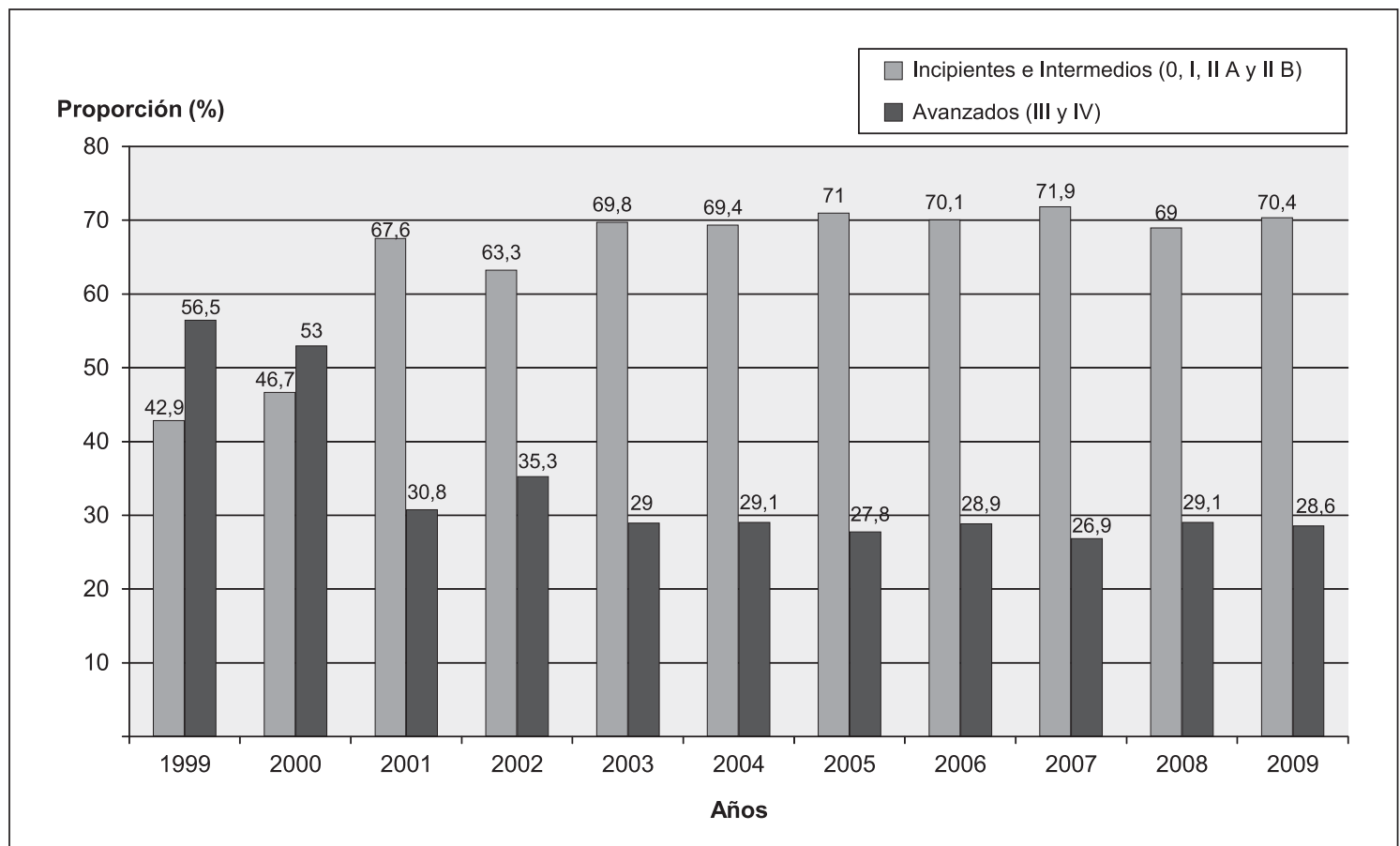

Figura 7. Incidencia proporcional de cánceres de mama según grado de avance (etapas fusionadas), Chile (SNSS), 19992009. Nota: no etapificado 0,4 a 1,0\% según año considerado. Algunos años no alcanzan el $100 \%$.

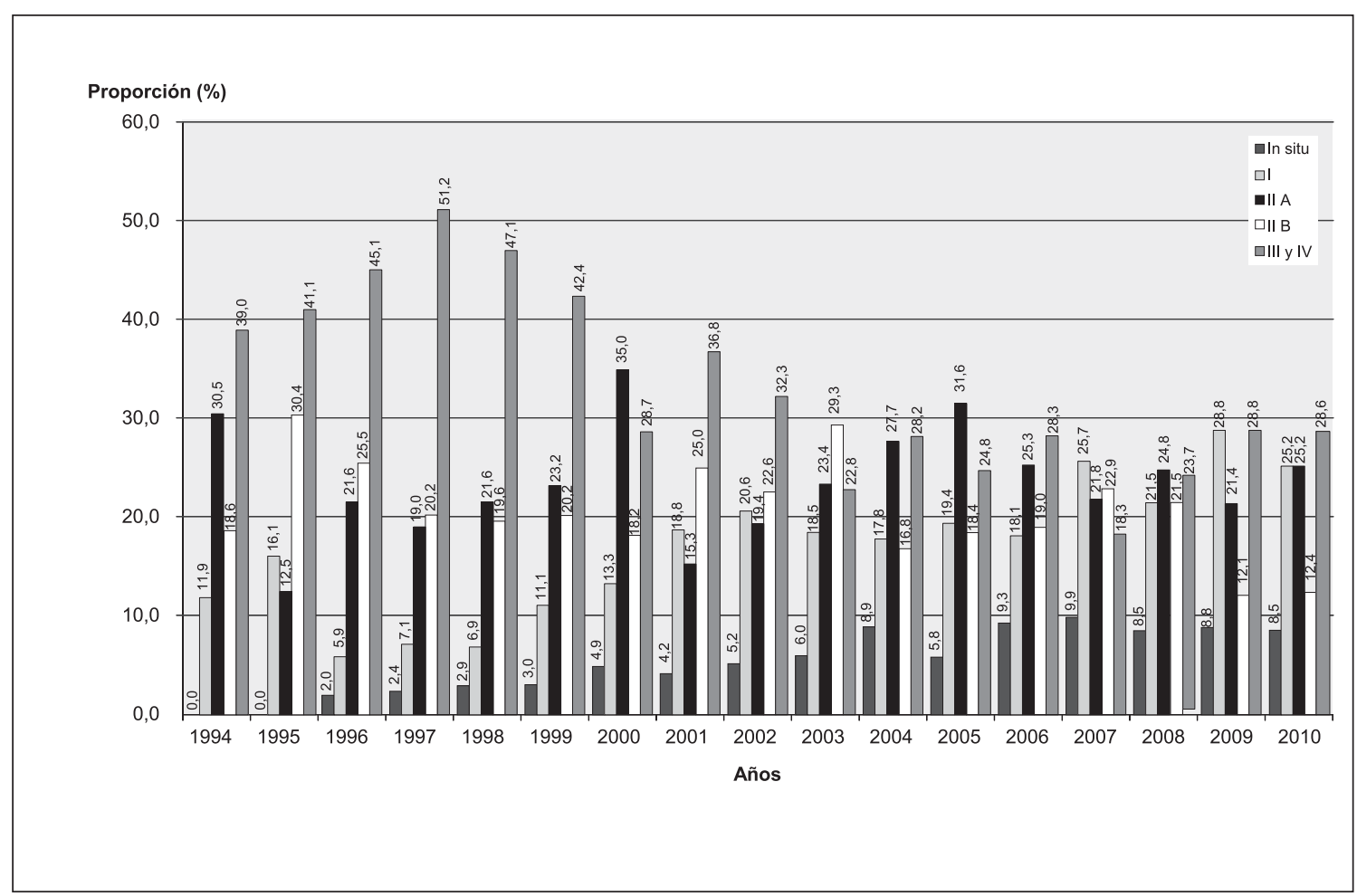

Figura 8. Incidencia proporcional de cánceres de mama según grado de avance: incipientes, intermedios y avanzados, SSMSO 1994-2010. 
estadíos avanzados, puede estar asociado a la preferencia etaria de mujeres jóvenes en el sector público. Creemos que la pérdida del trabajo en equipo entre hospitales y consultorios, junto con la no inclusión programática de mujeres sobre 54 años, pueden ser factores que juegan en contra de un diagnóstico más oportuno que signifique no tanto muchos estadíos 0 sino anticipación a estadíos III y IV, más frecuentes en senescentes, como se ha demostrado ${ }^{7}$. En base a la experiencia del SSMSO se puede proponer cambios en el programa nacional con participación de otros profesionales, además de matronas, para adelantarse a estadíos avanzados y captar un mayor número de mujeres de edades avanzadas, cuya tasa de mortalidad es 10 o 15 veces superior a la observada en mujeres jóvenes. Ambos factores: mayor edad y estadíos avanzados, se asocian con frecuencia según la experiencia internacional ${ }^{15}$. Algo diferente se observó en el único estudio chileno sobre cáncer de mama en mujeres senescentes, el cual mostró en una muestra pequeña que sobre 64 años existía sólo un $20 \%$ de estadíos III y IV ${ }^{16}$.

Nuestra propuesta que resulta de este estudio para una mayor captación de estas pacientes y adelantarse a estadíos avanzados, es aprovechar el enorme potencial de investigar cáncer de mama en pacientes con edades mayores, sobre 50 años, en los programas de diabetes mellitus e hipertensión arterial, así como en el examen de medicina preventiva del adulto y del adulto mayor, incorporando a las enfermeras que son el núcleo de la pesquisa de cáncer de mama en muchos países desarrollados ${ }^{17}$. Es posible que el hecho de no haber ocurrido así en nuestro programa nacional responda a una herencia de lo maternal y del cáncer cérvico uterino. Además, la responsabilidad programática descansa casi únicamente en matronas, las cuales tienen una formación académica que privilegia embarazo, parto y temas relacionados, además de captar en forma natural básicamente pacientes en edad fértil, con baja incidencia de cáncer mamario.

Creemos también que la tabulación de los cánceres de mama del SNSS debería desglosarse por grupos más segregados de edad. Un grupo de 35 a 64 y otro de 65 y más son demasiado grandes y significan una diversidad que es necesario conocer $^{18,19}$. Igualmente, es inadecuado en relación a grado de avance fusionar cuatro etapas con pronósticos tan diferentes como 0, I, II A y II B ${ }^{18,19}$. Se sugiere además que esa información sea publicada periódicamente para facilitar su análisis por grupos preparados e interesados en el tema.

El desarrollo de un registro nacional de cáncer en Chile se hace cada vez más imperativo ${ }^{20-24}$. En Chile sería muy fácil su desarrollo, al menos en dos cánceres que tienen programas nacionales como son cérvico uterino y mamario. Este tema, junto al rol del examen mamario como complemento al diagnóstico mamográfico, sea como tamizaje u oportunista, presenta abundantes publicaciones tanto en nuestro país como en muchos otros ${ }^{25-27}$. También se debe considerar el interés que presenta el estudio de la disminución de mortalidad por este cáncer y sus causas ${ }^{28-30}$. Entre nosotros falta una preocupación similar por su aumento y las razones del mismo. La disminución en los países desarrollados obedece a disminución de incidencia, mejores tratamientos y tamizaje mamográfico, el cual también presenta desventajas $^{31}$, por lo cual su promoción debe hacerse con un amplio debate y sólidos fundamentos ${ }^{32}$. Creemos que ha llegado el momento de cambiar nuestros paradigmas sobre cáncer de mama en Chile como ha ocurrido en otros países ${ }^{33}$.

\section{Agradecimientos}

Se agradece al Dr. Juan de Dios Reyes M., encargado de la Unidad de Cáncer en el Ministerio de Salud, período 2009-2010, su aporte de información sobre incidencia de cáncer de mama en el sector público de salud, 1999-2008. Esos datos nos han facilitado entender lo que está sucediendo con el cáncer de mama en Chile y permitido fundamentar propuestas para disminuir a futuro su creciente mortalidad.

\section{Referencias}

1. Fishler Y, Tarabeia J, Baron-Eppel O, Buchena M, Lipshitz I, Epala A. A comparison of trends in incidence and mortality of breast cancer. Eur J Cancer Prev. 2007;16:36-42.

2. Porter PL. Global trends in breast cancer incidence and mortality. Salud Publ Mex. 2009;51(suppl 2):S141S146.

3. Ministerio de Salud, Chile. Departamento de Estadística e Información en Salud (DEIS). Mortalidad por cáncer de mama femenina, 1999-2009. En: http://deis.minsal. cl/

4. Ministerio de Salud, Chile. Guía Clínica Examen Medicina Preventiva. Impreso, 2008. Cáncer de mama, pp.110-6.

5. Serra I, Martínez R, Mimica X, Cavada G, Aguayo C. Cáncer de mama en Chile. Un aporte clínico y epidemiológico según un registro poblacional metropolitano: 1.485 pacientes. Rev Chil Cir. 2009;60:507-14.

6. Serra I, Martínez R, Mimica X, Aguayo C. ¿Es posible disminuir la mortalidad por cáncer de mama en Chile? Análisis epidemiológico y programático en la Región Metropolitana, 2000-2007. Rev Chil Cir. 2010;62:33947. 
7. Serra I, Maya D, Martínez R. Cáncer de mama en Chile. Tardanzas en su atención médica. Cuad Med Soc. 2011;51:54-65.

8. Lozano-Ascencio R, Gómez-Dantés H, Lewis S, TorresSánchez L, López-Carrillo L. Salud Publ Mex. 2009; 51(suppl 2):S147-56.

9. Sant M, Aareleid T, Berrino F, Bielska LM, Carli PM, Faivre J, et al. EUROCARE-3: survival of cancer patients diagnosed 1990-1994 - results and commentary. Ann Oncol. 2003;14(suppl. 5):61-118.

10. Rodríguez J, Tokman M. Resultados y rendimiento del gasto en el sector público de salud en Chile 1990-1999. CEPAL, impreso, 56 pág.

11. Serra I, Calvo A, Sharp A. Perspectivas del cáncer biliar y otros cánceres importantes en Chile. Cuad Med Soc. 1988;29:126-33.

12. Serra I. ¿Ha disminuido la mortalidad por cáncer vesicular en Chile? Rev Med Chile 2001;129:1079-84.

13. Andía M, Gederlini A, Ferreccio C. Cáncer de vesícula biliar: tendencia y distribución del riesgo. Rev Med Chile 2006;134:565-74.

14. Peralta O. Cáncer de mama en Chile. Epidemiología y factores de riesgo. Cuad Med Soc. 2007;47:18-30.

15. Paesmans M, Ameye L, Moreau M, Rozenberg S. Breast cancer screening in the older woman: An effective way to reduce mortality? Maturitas 2010;66:263-7.

16. Escobar P, Camacho J, Barriga C, Toledo K, Brucher R. Cáncer de mama en pacientes mayores de 65 años. Influencia de la edad en la presentación y manejo. Rev Chil Cir. 2003;55:476-9.

17. Chiarelli AM, Machpruz V, Brown P, Theriault M, Edwards S, Shumak R, et al. Influence of nurses on compliance with breast cancer screening recommendations in an organized breast cancer screening program. Cancer Epidemiol Biomarkers Prev. 2010;19:697-706.

18. Prieto M, Torres S. Situación epidemiológica del cáncer de mama en Chile, 1994-2003. Rev Med Clin Condes 2006;17:142-8.

19. Reyes Jd D. Comunicación personal, 2009.

20. Vallebuona C. Registros poblacionales de cáncer. Avances en Chile. El Vigía 2001;14:12.
21. Roa I. La necesidad de crear registros de cáncer. Rev Chil Cir. 2002;54:206-11.

22. Rutqvist L, Wallgren A. Inconsistencies in breast carcinoma registration. An investigation of 855 cases reported by the Swedish Cancer Registry. Acta Radiol Oncol. 1983;22:109-12.

23. Edwards D, Bell J. Cancer registries-future development and uses in Britain. J Publ Health Med. 2000;22:216-9.

24. Hery C, Ferlay J, Boniol M, Autier P. Changes in breast cancer incidence and mortality in middle-aged and elderly women in 28 countries with Caucasian majority populations. Ann Oncol. 2008;19:1009-18.

25. Escobar P, Herrera R. Auto examen mamario: su aporte en el diagnóstico precoz del cáncer de mama. Rev Chil Obstet Ginecol. 2000;65:407-11.

26. Miller AB. Practical applications for clinical breast examination (CBE) and breast self-examination (BSE) in screening and early detection of breast cancer. Breast Care. 2008;3:17-20.

27. Miller AB, Baines CJ. The role of clinical breast and breast self-examination. Prev Med. 2011. En: doi:10. 1016/j.yomed.2011.05.00.

28. Beral V, Hennon C, Reeves G, Peto. Sudden fall in breast cancer death rates in England and Wales: Lancet. 1995;345:1642-3.

29. Peto R, Boreham H, Clarke M, Dayles C, Beral V. UK and USA breast cancer deaths down 25\% at ages 20-69 years. Lancet 2000;355(9217):1522.

30. Richards MA, Stockton D, Babb P, Coleman MP. How many deaths have been avoided through improvement in cancer survival? BMJ 2000;320:395-8.

31. Elmore JG, Armstrong K, Lehman CD, Fletcher SN. Clinical review: Screening for breast cancer. JAMA 2005;293:1089-95.

32. Puschel K, Thompson B. Mammogram screening in Chile: Using mixed methods to implement health policy planning at the primary care level. Breast 2011;20:S40S45.

33. Miller AB. Screening for breast cancer: is it time for a paradigm shift? Ann Royal College of Physicians and Surgeons of Canada 1994;27:353-5. 